\title{
CONSTRUÇÃO DE UMA BOMBA PERISTÁLTICA E DE UM SISTEMA DE DETECÇÃO UTILIZANDO UM HARDWARE DE CÓDIGO FONTE ABERTO “ARDUINO” PARA ANÁLISE EM FLUXO
}

\author{
Fernanda S. C. Soares , Alan L. Vieira ${ }^{\mathrm{b}}$, Márcio S. Soares ${ }^{\mathrm{a}}$, Marcus V. Sant'Anna ${ }^{\mathrm{a}}$, Rejane Nascentes ${ }^{\mathrm{a}}$ e Carla M. Bossü** \\ anstituto de Ciências Exatas e Tecnológicas, Universidade Federal de Viçosa Campus Rio Paranaíba, 38810-000 Rio Paranaíba \\ - MG, Brasil \\ 'Instituto de Química, Universidade Estadual Paulista Júlio de Mesquita Filho, 14800-060 Araraquara - SP, Brasil
}

Recebido em 02/05/2018; aceito em 29/08/2018; publicado na web em 18/09/2018

\begin{abstract}
PERISTALTIC PUMP AND DETECTION SYSTEM CONTROLLED BY AN ARDUINO OPEN SOURCE HARDWARE DEVELOPED AND DESIGNED FOR APPLICATION IN A FLOW ANALYSIS SYSTEM. This work describes the development of a peristaltic pump and a photometric detector, using "Arduino" and alternative and low-cost materials for flow analysis system. The analytical calibration curve of the acid-red-283 using the proposed system presents 0.9984 and $3.9 \%$ as the coefficients of determination and variation, and 1.1 and $3.8 \mathrm{mg} \mathrm{L}^{-1}$ as limits of detection and quantification, respectively. Three prepared samples of the acid-red-283 (25, 30 and $\left.35 \mathrm{mg} \mathrm{L}^{-1}\right)$ showed recovery between 99 to $104 \%$. For construction of the peristaltic pump and the photometer using Light Emitting Diodes (LED), light-dependent resistor (LDR) were spent less than 10\% of the cost was spent on a commercial system employing a peristaltic pump and a spectrophotometer. Furthermore, the peristaltic pump and the photometer developed in this work allow the implementation of practical disciplines, such as molecular absorption spectrophotometry and flow analysis system in undergraduate courses, making these instruments more accessible, especially for the chemistry students.
\end{abstract}

Keywords: flow analysis; Arduino; peristaltic pump.

\section{INTRODUÇÃO}

O avanço tecnológico resultante do severo controle de qualidade nas indústrias vem influenciando a química analítica e os métodos clássicos de análise. A necessidade de informações de qualidade em tempo real abriu o precedente para o desenvolvimento de uma nova geração de microdispositivos integrados a diferentes etapas de uma análise química. ${ }^{1}$

Os sistemas de análise em fluxo possibilitam uma maneira flexível e simples de conectividade, parcial ou total, de diferentes etapas de uma análise química. ${ }^{2}$ Os sistemas em fluxo trouxeram um avanço significativo em química analítica, a facilidade em se controlar o tempo do procedimento a partir de dispositivos eletrônicos e acoplar detectores controlados por microcomputadores permitiu que os métodos assumissem características automáticas. ${ }^{3}$

A propulsão dos fluidos nesses sistemas pode ser feita por ação da gravidade, por propulsão a gás, bomba de pistão ou ainda pela utilização de uma bomba peristáltica. ${ }^{4} \mathrm{~A}$ bomba peristáltica geralmente é mais utilizada, uma vez que permite a vazão constante das soluções e robustez na operação. O bombeamento através do sistema peristáltico não provoca o mínimo dano ao fluido e é efetuado sem qualquer contaminação. Existem bombas com número variável de canais, ou seja, suportes para o acoplamento dos tubos Tygon ${ }^{\circledR}$ por onde as soluções serão transportadas e a velocidade da propulsão dos fluidos pode ser ajustada na bomba peristáltica e o fluxo transportado vai depender do diâmetro do tubo utilizado. Entre as limitações de uso da bomba peristáltica, pode-se destacar, principalmente, o custo elevado do próprio equipamento. ${ }^{5}$

Além disso, inúmeros detectores têm sido empregados nas análises em fluxo, dentre os quais espectrofotômetros UV-VIS, espectrofotômetros de absorção atômica por chama, potenciômetros e condutivímetros. ${ }^{6}$ Os diodos emissores de luz (light emitting diodes - LED) vêm sendo amplamente empregados como fontes de radiação

*e-mail: carla.bossu@gmail.com na construção de equipamentos espectrofotométricos portáteis, devido ao fato de se tratar de componentes de baixo custo, simples e com dimensões reduzidas. Os LEDs emitem radiação em uma estreita faixa de comprimento de onda, sendo possível encontrá-los em diferentes cores, desde o violeta até o infravermelho próximo. O emprego de LED como fonte de radiação dispensa a utilização de lâmpadas incandescentes (tungstênio) e dispositivos ópticos, lentes, filtros e prismas, possibilitando construir fotômetros simples, de baixo custo, duráveis e de pequeno porte. ${ }^{7,8}$

No que se refere à aquisição de sinal analítico, alternativas de baixo custo esbarram na dificuldade em construir circuitos eletrônicos e no desenvolvimento de softwares, uma vez que a maioria dos analistas não possui formação nessa área. Neste caso, uma alternativa a essa limitação é o uso da plataforma comercial "Arduino UNO”, plataforma de hardware de código fonte aberto com comunicação serial USB, de fácil aquisição, baixo custo e com software livre. Existem diferentes modelos comerciais de "Arduino", que podem possuir de 14 a 54 pinos digitais de entrada e saída de dados, os quais podem ser utilizados para controle de dispositivos, tais como bomba peristáltica, válvulas e bombas solenoide, além de fazer a aquisições de dados..$^{9,10}$

Neste contexto, o objetivo desta nota técnica foi desenvolver um sistema em fluxo utilizando materiais alternativos e de baixo custo para construção especialmente de uma bomba peristáltica e, de forma complementar, fez-se o desenvolvimento de um fotômetro de absorção molecular. O sinal analítico do fotômetro desenvolvido foi obtido diretamente em um laptop através do uso de um "Arduino UNO", um hardware de código fonte aberto. A potencialidade de aplicação do sistema proposto foi comprovada pela análise do azo corante acid-red-283 em água, obtendo resultados satisfatórios quando comparado a um sistema comercial empregando análise em batelada. No desenvolvimento do fotômetro, utilizou-se um LED azul de alto brilho como fonte de radiação, uma célula de fluxo com caminho óptico de $1 \mathrm{~cm}$ feita de acrílico e como detector um resistor dependente de luz (LDR), os quais foram instalados no interior de uma caixa preta de polipropileno. Para aquisição dos dados do LDR 
diretamente em um computador foi empregado um microcontrolador "Arduino". A principal inovação foi a construção da bomba peristáltica de baixo custo, que juntamente com a utilização do "Arduino" em conjunto com LDR, possibilitou a comunicação com o computador, melhorando a aquisição dos dados, permitindo, assim, uma análise em fluxo robusta, mesmo se tratando de um equipamento alternativo.

\section{PARTE EXPERIMENTAL}

\section{Instrumentação}

Para a validação dos resultados obtidos neste trabalho foi utilizado o Espectrofotômetro de UV-Vis (Thermo Evolution 300).

\section{Sistema em fluxo}

Uma válvula de injeção rotacional ${ }^{11}$ foi utilizada para injeção da amostra. A alça de amostragem foi otimizada, sendo o volume de $780 \mu \mathrm{L}$ utilizando para melhor obtenção dos sinais transientes dos padrões e amostras.

As análises em fluxo empregando o fotômetro desenvolvido foram realizadas empregando uma cela de fluxo construída de acrílico com caminho óptico de $1 \mathrm{~cm}$.

Tubos Tygon ${ }^{\circledR}(\mathrm{d} . \mathrm{i}=0,8 \mathrm{~mm})$ foram utilizados na bomba peristáltica e tubos de polietileno $(\mathrm{d} . \mathrm{i}=0,5 \mathrm{~mm})$ foram usados como percurso analítico do sistema em fluxo.

\section{Construção da bomba peristáltica}

Em análise em fluxo, as bombas peristálticas são as mais utilizadas para a movimentação dos fluidos, por apresentarem boa reprodutibilidade e precisão no fluxo. Porém, apresentam custo relativamente elevado, razão pela qual optou-se pela construção de uma bomba peristáltica com materiais alternativos e de baixo custo.

Para a construção da bomba peristáltica foram empregados os seguintes materiais: um motor de vidro elétrico automotivo (12 V); um suporte para fixar o motor; cinco cilindros de cloreto de polivinila (PVC) com $10 \mathrm{~cm}$ de comprimento e 31,75 mm de diâmetro interno; dez rolamentos $\left(\mathrm{N}^{\circ}\right.$ 6002) com diâmetro externo de 32 $\mathrm{mm}$ e duas molas com $10 \mathrm{~cm}$ de comprimento. Para a montagem e fixação das peças foram utilizados parafusos de " $3 / 16$ ". Todos esses materiais foram adquiridos em oficinas mecânicas e em comércio para materiais de construção civil. Para ajuste da velocidade de rotação do motor empregou-se um potenciômetro na saída da fonte de alimentação.

Os roletes da bomba peristáltica foram feitos torneando os cilindros de PVC de modo que os rolamentos encaixassem facilmente em suas extremidades. Na bomba peristáltica foram utilizados cinco roletes, os quais foram conectados ao eixo central através de duas chapas de aço (d.i $=10 \mathrm{~mm}$ ) e cinco parafusos (3/16") de $11,5 \mathrm{~cm}$ de comprimento, como pode ser observado na Figura 1. O eixo central foi construído utilizando uma barra rosqueada (5/8") com $23 \mathrm{~cm}$ de comprimento conectada ao motor em uma extremidade e na outra em um suporte contendo um rolamento ( $\mathrm{N}^{\circ}$ 6002). Para fixar os tubos Tygon ${ }^{\circledR}$ na bomba peristáltica foram utilizadas duas chapas de ferro $(11 \times 2,5 \mathrm{~cm})$ com 3 fendas $(10 \times 2 \mathrm{~mm})$. Uma chapa metálica dobrada em formato de meia circunferência $(\mathrm{r}=10 \mathrm{~cm})$ foi utilizada para fazer a tampa superior da bomba peristáltica, a qual foi pressionada contra os roletes através de 2 molas. Dois guias foram feitos para impedir que a tampa da bomba peristáltica deslocasse lateralmente. Uma placa de madeira laminada $(43 \times 30 \mathrm{~cm})$ foi utilizada para sustentação de todas as peças.

Uma fonte de corrente contínua de $12 \mathrm{~V}$ e 10 A foi utilizada como fonte de alimentação do motor. Um potenciômetro foi adaptado a essa fonte a fim de controlar a velocidade do motor e, consequentemente, o fluxo.

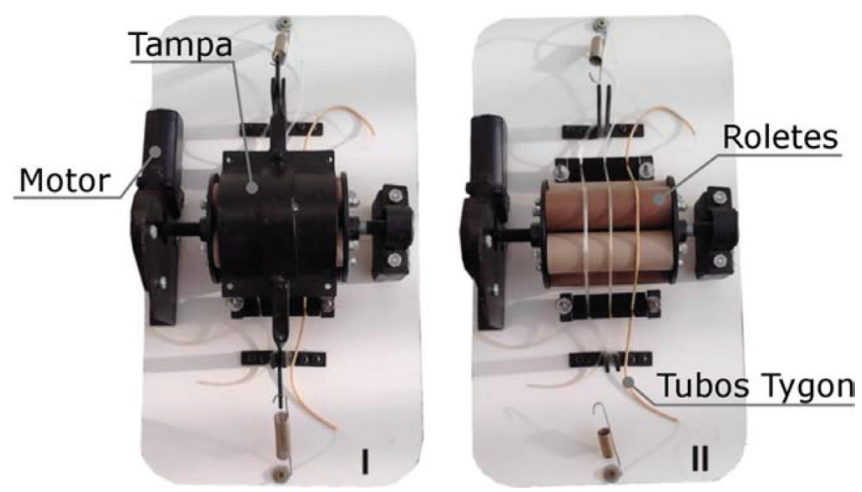

Figura 1. Bomba peristáltica; (I) com a tampa superior; (II) sem a tampa superior

\section{Construção do fotômetro e aquisição do sinal analítico}

Para a construção do fotômetro alternativo foi utilizada uma caixa de ABS (acrilonitrila butadieno estireno) de coloração preto fosco com dimensões de $5,5 \mathrm{~cm}$ de altura, $4,5 \mathrm{~cm}$ de largura e $8 \mathrm{~cm}$ de comprimento. Uma lâmpada LED e um resistor dependente de luz (LDR) foram empregados como fonte de radiação e detecção, respectivamente. $\mathrm{O}$ fotômetro desenvolvido pode ser utilizado em modo batelada ou em fluxo, sendo necessário apenas a troca da cela de análise. Para análise em fluxo foi desenvolvido uma cela de acrílico com caminho ótico de $1 \mathrm{~cm}$. As análises em batelada foram conduzidas utilizando cubetas de acrílico descartáveis. Uma ilustração do fotômetro desenvolvido está apresentada na Figura 2.
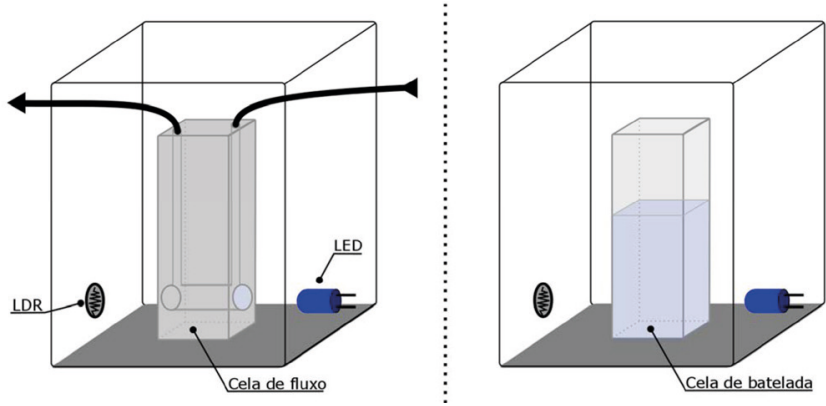

Figura 2. Ilustração do fotômetro desenvolvido; Modo de análise em fluxo (esquerda) e modo de análise em batelada (direita)

Para a obtenção do sinal analítico foi utilizado um micro controlador Arduino, responsável por captar o sinal analógico do LDR e enviá-lo para o microcomputador na forma de sinal digital. $\mathrm{O}$ circuito eletrônico que conecta o LDR ao micro controlador Arduino encontra-se exemplificado na Figura 3. Para que o Arduino execute a função de obter o sinal do LDR e exportá-lo para o microcomputador foi utilizado o programa apresentado na Tabela 1.

Os sinais registrados pelo programa são, então, transferidos para uma planilha para a construção das curvas analíticas e cálculos das concentrações.

\section{Aplicação do fotômetro para a quantificação de azo corantes}

A solução estoque de $50 \mathrm{mg} \mathrm{L}^{-1}$ do azo corante acid-red-283 $\left(\right.$ Luganil $\left.^{\circledR}\right)$ foi preparada a partir da diluição de $50 \mathrm{mg}$ do corante em 


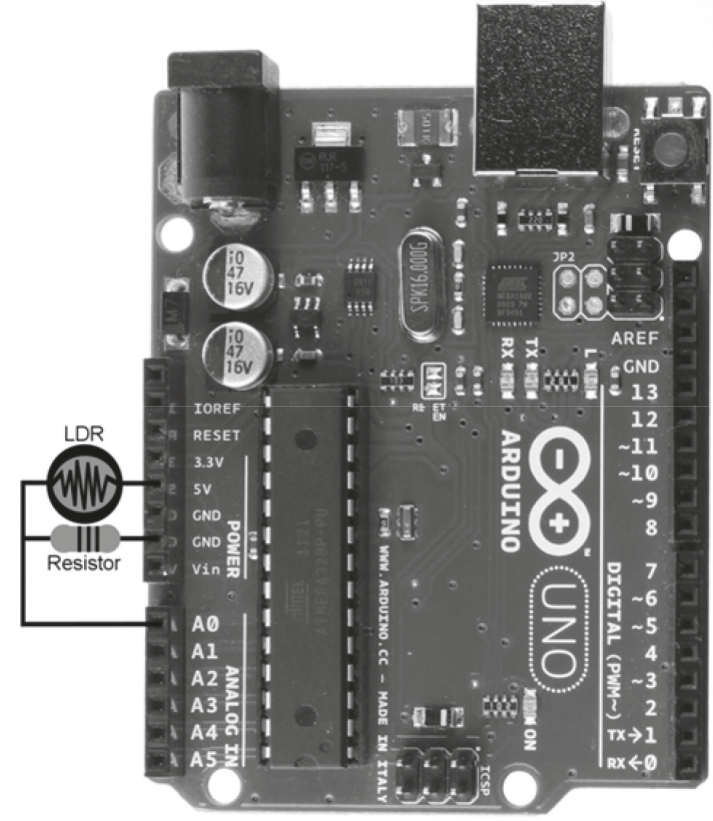

Figura 3. Circuito eletrônico-LDR e Arduino. LDR-3 mm e resistor de $10 \mathrm{k} \Omega$

Tabela 1. Programação do Arduino para aquisição do sinal do LDR. Os comentários da função de comando em itálico e precedido de "//"

Funções para aquisição do sinal do LDR pelo Arduino

int valor $=0$;

void $\operatorname{setup}()$

\{Serial.begin(115200);

void $\operatorname{loop}()$

$\{$ valor $=$ analogRead $(0)$;

Serial.println(valor); $\quad$ // registra o sinal analógico da porta “ 0 ” (LDR). delay(1000); $\} \quad$ // tempo entre cada valor registrado $(1000 \mathrm{~ms})$.

um balão volumétrico de $1000 \mathrm{~mL}$ e avolumados com água purificada em sistema Milli-Q (18,2 M $\Omega \mathrm{cm})$.

A curva analítica até $40.0 \mathrm{mg} \mathrm{L}^{-1}$ e três amostras $(25,30$ e 35 $\mathrm{mg} \mathrm{L}^{-1}$ ) do azo corante acid-red-283 foram preparadas a partir da diluição da solução estoque de $500 \mathrm{mg} \mathrm{L}^{-1}$ previamente preparada. Tanto as amostras como as soluções padrão para a curva analítica foram preparadas em quintuplicata $(n=5)$.

As leituras realizadas no fotômetro desenvolvido foram conduzidas utilizando um LED Azul de alto brilho $\left(\lambda_{\text {máx }}=470 \mathrm{~nm}\right)$, visto que o azo corante acid-red-283 apresenta uma banda de absorção em $501 \mathrm{~nm}$.

As soluções foram analisadas em batelada e em fluxo pelo fotômetro proposto, sendo o sinal da leitura menos o sinal do branco adotado no modo batelada e a intensidade máxima do pico transiente utilizado para análise em fluxo. A bomba peristáltica proposta foi utilizada para análises em fluxo, de acordo com o diagrama apresentado na Figura 4. Para reduzir a pulsação no fluxo da bomba peristáltica foi utilizado como amortecedor de pulsação uma seringa de $5 \mathrm{~mL}$. $\mathrm{O}$ amortecedor de pulsação contém um volume morto preenchido de ar, o qual atua sobre a pulsação da bomba peristáltica e minimiza a pulsação no fluído. $\mathrm{O}$ amortecedor de pulsação é utilizado apenas na via do fluído transportador.

Para fins comparativos, um espectrofotômetro comercial (Thermo Evolution 300 UV-Vis) foi utilizado para análise em batelada do azo corante acid-red-283.

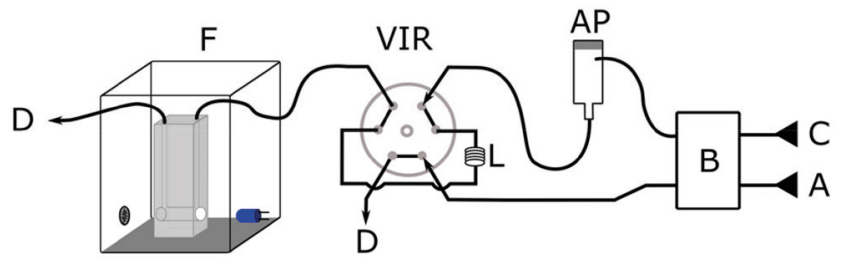

Figura 4. VIR - Válvula de injeção rotacional; L: alça de amostragem $(780 \mu L)$; $C$ : fluido transportador

\section{RESULTADOS E DISCUSSÃO}

A bomba peristáltica desenvolvida conta com um potenciômetro para controlar a velocidade de rotação do motor e, por conseguinte a velocidade do fluxo. Para verificar a estabilidade do fluxo produzido pela bomba peristáltica foi interpolado um gráfico de volume por tempo ao ligar a bomba peristáltica e após três horas de funcionamento contínuo. Para tanto, foi considerado o tempo gasto para a propulsão de $5,10,15,20$ e $25 \mathrm{~mL}$ da solução transportadora pelo percurso analítico $(150 \mathrm{~cm})$. A equação da reta e o fluxo médio obtidos nas duas situações estão apresentadas na Tabela 2, demonstrando que as equações lineares obtidas em ambas situações foram muito próximas. Além disso, os fluxos médios não apresentaram diferenças significativas pelo teste $t$ de Student, a 95\% de confiança. A bomba peristáltica e a fonte de alimentação não sofreram um aquecimento considerável durante as três horas contínuas de uso, demonstrando boa reprodutibilidade.

A análise do azo corante acid-red-283 foi realizada nos modos batelada e em fluxo e os resultados comparados com o espectrofotômetro comercial, sendo as curvas resultantes apresentadas na Figura 5.

Pela análise da Figura 5 nota-se que os três modos de análise forneceram calibrações com coeficientes de correlação próximos de 1 , demonstrando forte correlação entre os eixos. O desvio padrão relativo na curva obtida pelo espectrofotômetro de UV-Vis, fotômetro em batelada e em fluxo foram de $1 \%, 3 \%$ e $6 \%$, respectivamente. Como pode ser observado na Tabela 3, os limites de detecção (LOD) e quantificação (LOQ) do espectrômetro UV-Vis e do fotômetro em batelada foram da mesma ordem de magnitude, no entanto, para o fotômetro em fluxo, o limite de detecção e quantificação foram da ordem de dez vezes superiores devido aos diferentes arranjos experimentais, à maior diluição das amostras e reagentes e se tratar de medida cinética, diferente das condições em estado estacionário. Vale ressaltar que as medidas empregando dispositivo "Arduino" foram registradas como sinal analítico, compreendido entre 0 (forte incidência de luz sobre o LDR) e 1024 (ausência de luz sobre o LDR).

Para avaliar a exatidão e precisão das determinações, três amostras do azo corante acid-red-283 (25, 30 e $\left.35 \mathrm{mg} \mathrm{L}^{-1}\right)$ foram preparadas pela diluição da solução estoque. Na Tabela 4 são apresentados os valores obtidos quando analisadas as diferentes concentrações preparadas do azo corante $\left(25,30\right.$ e $\left.35 \mathrm{mg} \mathrm{L}^{-1}\right)$. Os resultados foram

Tabela 2. Controle do fluxo em relação ao tempo de funcionamento da bomba peristáltica

\begin{tabular}{|c|c|c|c|}
\hline Tempo de funcionamento & Equação da reta & $\mathrm{R}^{2}$ & Fluxo médio (mL/min) \\
\hline Início & $y=0,1616 x-0,016$ & 0,9998 & $6,26 \pm 0,09$ \\
\hline Após três horas & $y=0,1628 x-0,026$ & 0,9997 & $6,3 \pm 0,1$ \\
\hline
\end{tabular}



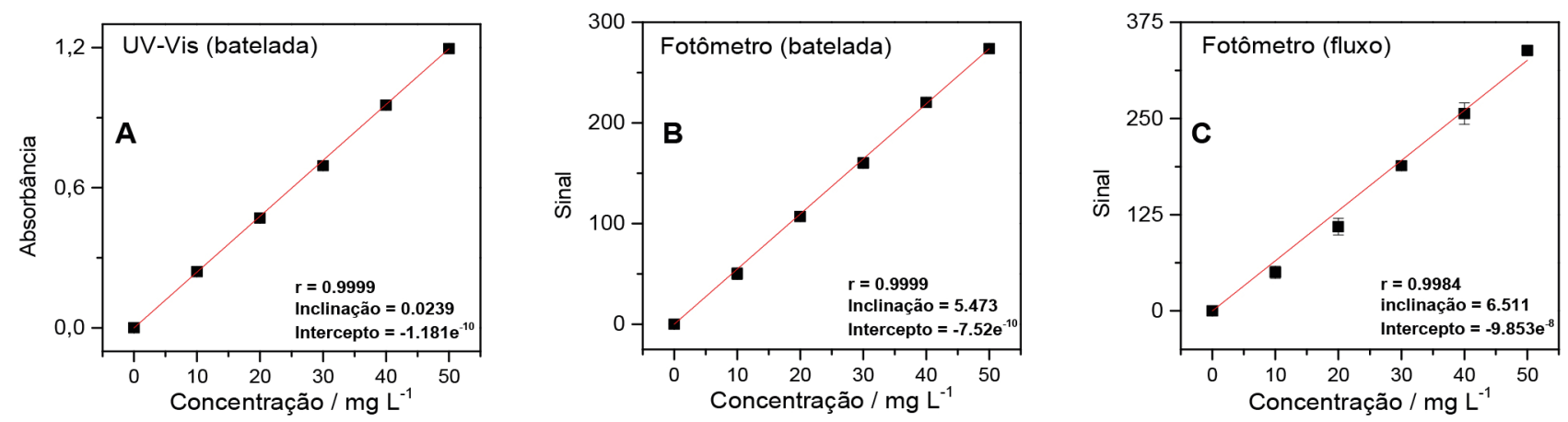

Figura 5. Curvas de calibração para o azo corante acid-red-283. A - Espectrômetro UV-Vis; B - Fotômetro em batelada; C - Fotômetro em fluxo

Tabela 3. Valores dos limites de detecção (LOD) e dos limites de quantificação (LOQ)

\begin{tabular}{lcc}
\hline Equipamentos & $\mathrm{LOD}\left(\mathrm{mg} \mathrm{L}^{-1}\right)$ & $\mathrm{LOQ}\left(\mathrm{mg} \mathrm{L}^{-1}\right)$ \\
\hline UV-Vis comercial & 0,12 & 0,40 \\
Fotômetro em batelada & 0,11 & 0,36 \\
Fotômetro em fluxo & 1,13 & 3,76
\end{tabular}

$\mathrm{LOD}=3 \times \mathrm{SD}_{\mathrm{bco}}$ /inclinação; $\mathrm{LOQ}=3,33 \times \mathrm{LOD} ; \mathrm{SD}_{\mathrm{bco}}=$ desvio padrão dos brancos $(\mathrm{n}=10)$.

comparados e não se mostraram estatisticamente diferentes ao nível de $95 \%$ de confiança (teste $t$ Pareado). Os desvios padrão relativos obtidos pelo fotômetro em batelada e em fluxo foram respectivamente da ordem de 1,7 e $3 \%$. No que se refere às análises empregando o espectrômetro de UV-Vis comercial, o desvio padrão relativo foi da ordem de $0,8 \%$. O fotômetro, tanto operado em modo batelada quanto em fluxo apresentou desempenho satisfatório, proporcionando boa exatidão e precisão quando comparado com o espectrômetro UV-Vis comercial e os valores de desvio padrão relativo foram baixos e estão concordantes com os valores aceitáveis na literatura. ${ }^{12,13}$

Tabela 4. Concentrações $\left(\mathrm{mg} \mathrm{L}^{-1}\right)$ obtidas $(n=5)$ pelo espectrofotômetro UV-Vis comercial, fotômetro em batelada e em fluxo nos três níveis de diluição

\begin{tabular}{cccc}
\hline \multirow{2}{*}{$\begin{array}{c}\text { Concentração } \\
\text { preparada }\end{array}$} & \multicolumn{3}{c}{ Concentração determinada } \\
\cline { 2 - 4 } & $\begin{array}{c}\text { UV-Vis } \\
\text { comercial }\end{array}$ & $\begin{array}{c}\text { Fotômetro } \\
\text { em batelada }\end{array}$ & $\begin{array}{c}\text { Fotômetro } \\
\text { em fluxo }\end{array}$ \\
\hline 25 & $25,0 \pm 0,3$ & $25,2 \pm 0,4(0,69)$ & $24,9 \pm 1(0,17)$ \\
30 & $29,8 \pm 0,2$ & $30,1 \pm 0,6(1,50)$ & $29,2 \pm 0,9(2,06)$ \\
35 & $34,8 \pm 0,2$ & $35,1 \pm 0,5(1,76)$ & $35,3 \pm 0,7(2,17)$ \\
\hline
\end{tabular}

Valor de $t$ pareado com 95\% de confiança $=2,78 ;()=$ valor de $t$ calculado.

A bomba peristáltica proposta possibilitou a análise em fluxo com baixo custo, sendo este modo de análise condizente com os princípios da química verde, haja vista que são utilizados menos reagentes e requer menor tempo de análise. É importante ressaltar que a utilização do "Arduíno" foi útil para registrar os sinais obtidos pelo LDR, principalmente quando se refere à análise em fluxo, devido à velocidade para registrar o sinal transiente característico destas análises. Com a utilização do "Arduino" foi possível construir um fotômetro tanto em batelada quanto em fluxo, mantendo o baixo custo e simplicidade operacional. Deste modo, a aquisição dos dados empregando "Arduino" possibilita uma análise mais robusta mesmo se tratando de um equipamento alternativo.

\section{CONCLUSÃO}

Neste trabalho, uma bomba peristáltica e um fotômetro para análise em batelada e em fluxo foram desenvolvidos empregando materiais alternativos e de baixo custo. Os resultados obtidos demonstram que a bomba peristáltica construída pode ser utilizada como alternativa às bombas peristálticas comerciais.

O sistema de aquisição dos dados diretamente em um microcomputador durante a análise em batelada e em fluxo foi desenvolvido empregando um hardware "Arduino" em conjunto com LDR, que possibilitou a obtenção de resultados comparáveis com um espectrômetro UV-Vis comercial.

Em relação aos custos, para a construção da bomba peristáltica foram gastos aproximadamente US\$125,00, valor bem mais acessível do que uma bomba comercial ( US\$ 3.900,00). Outro fato relevante é o baixo custo inerente à construção deste fotômetro alternativo, para o qual foi gasto US\$37,00, sendo este valor muito inferior aos espectrofotômetros mais simples encontrados no mercado ( US\$ $1.700,00)$.

Além disso, a bomba peristáltica e o fotômetro desenvolvidos neste trabalho podem ser utilizados em disciplinas práticas como a espectrofotometria de absorção molecular e análise em fluxo em cursos técnicos e de graduação em química, com instrumentos mais acessíveis aos alunos.

\section{AGRADECIMENTOS}

Ao Programa PIBEN da Universidade Federal de Viçosa Campus Rio Paranaíba

\section{REFERÊNCIAS}

1. Martínez-Cisneros, C. S.; Ibáñez-García, N.; Valdés, F.; Alonso, J.; Sens. Actuators, A 2007, 138, 63.

2. Rocha, F. R. P.; Martelli, P. B.; Reis, B. F.; Quim. Nova 2000, 23, 119.

3. Zagatto, E. A. G.; Oliveira, C. C.; Collins, C. H.; Quim. Nova 1999, 22, 143.

4. Holler, F. J.; Skoog, D. A.; Crouch, S. R.; Princípios de Análise Instrumental, 6 ed.; Bookman: Porto Alegre, 2009.

5. Matos, R. C.; Gutz, I. G. R.; Angnes, L.; Fontenele, R. S.; Pedrotti, J. J.; Quim. Nova 2001, 24795.

6. Reis, B. F.; Zagatto, E. A.; Jacinto, A. O.; Krug, F. J.; Bergamin, F. H.; Anal. Chim. Acta 1980, 119.

7. Veras, G.; Silva, E. C.; Lyraa, W. S.; Soares, S. F. C.; Guerreiro, T. B.; Santos, S. R. B. A.; Talanta 2009, 77, 1155.

8. Dias,T.; R.; Brasil, M.; A.; S.; Sens. Actuators, B 2014, 198, 448.

9. Gonzáles, P.; Pérez, N.; Knochen, M.; Quim. Nova 2016, 3, 305.

10. Kamogawa, Y. K.; Miranda, C. J.; Quim. Nova 2013, 36, 1232.

11. Kolev, S. D.; McKelvie, I. D.; Compr. Anal. Chem. 2008, 54, 777.

12. Taverniers, I.; Loose, M. D.; Bockstaele, E. V.; Trends Anal. Chem. 2004, 23,535 .

13. Chudzinka, M.; Debska, A.; Danuta, B.; Accredit. Qual. Assur. 2011, 16, 1. 